\title{
PHYSICOCHEMICAL PARAMETERS OF PROBIOTIC YOGHURT WITH BIOACTIVE NATURAL PRODUCTS FROM SEA BUCKTHORN
}

\author{
- research paper -

\begin{abstract}
Anca TIFREA *11 , Ovidiu TIŢA* , Endre MÁTHÉ** , Otto KETNEY*
* "Lucian Blaga” University of Sibiu, Faculty of Agricultural Sciences, Food Industry and Environment Protection, Department of Food

Biotechnology, Dr. Ion Ratiu Street, no. 7-9, 550024 Sibiu, Romania
\end{abstract} \\ **2Genetics and Molecular Biology Research Group, Institute of Biology \\ and Environmental Sciences, University College of Nyiregyhaza, Sostoi ut \\ 31/B, Nyiregyhaza 4400, Hungary
}

\begin{abstract}
Probiotic dairy products are part of functional foods, which are made from fermented milk selected from a culture or a concentrate of bacteria that improve the microbial balance in the gut. These products have positive and beneficial influence on health and integrity of the human body. In the present study regarding the fortification with oil and pulp of sea buckthorn, was to obtained product similar to the conventional yoghurt, consistency and texture aimed at reducing heat treatments and stages technology in order to preserve nutritional quality. This research focused on optimizing the technology for obtaining yoghurt and selecting the best options regarding the addition of sea buckthorn pulp or oil, based on the following parameters : acidity, $\mathrm{pH}$ and lactic acid. At the same time we aimed to obtain probiotic yoghurt to make comparative studies on major parameters and to select the optimal probiotic yoghurt with sea bucktorn that give a good stabilization.
\end{abstract}

Keywords: probiotic yoghurt,lactic acid,sea buckthorn,acidity,bioactive natural products

${ }^{1}$ Corresponding author. Mailing adress: Mailing address: University "Lucian Blaga" of Sibiu, Faculty of Agricultural Sciences, Food Industry and Environmental Protection, 7-9 Ion Raţiu street, 550012 Sibiu, Romania. Phone: 0040/269/211338. Fax: 0040269212558 , E-Mail: tifreaanca@yahoo.com

Vol. XVII (2013), no. 1 


\section{INTRODUCTION}

Functional food is a term that is being used to define the foods which provide bioactive natural compounds to human diet beyond their basic nutrient supplying characteristics (Karaaslan et al., 2011). These bioactive compounds are often subject to health - benefiting and disease - preventing claims (Kris-Etherton et al., 2004).

Sea buckthorn juice contains high concentrations of vitamin $\mathrm{C}$ (Beveridge et al., 2002). The juice derived from the fruit is high in vitamin $\mathrm{C}$ and carotenes, which provides for the bright orange color (Beveridge et al., 1999). The $\mathrm{pH}$ of the juice is near 2.5 , wich is a lower value than in most common temperate fruits (Beveridge et al., 2002) .

Sea buckthorn leaves contain nutrients and bioactive substances. These include flavonoid (Chen, 1991), carotenoids, free and esterified sterols, triterpenols, and isoprenols(Goncharova and Glushenkova, 1996).

The food for health named also - Food for Specific Health Uses (FOSHU) are foodstuff containing biologically active products, obtained from raw material with high biologic potential. These biologic products consumed with current food, contribute to maintain the optimum state of physical and mental health of the population (Hasler, 1998).

Lankaputhra and Shah (1997) studied the effect of two stage fermentation on the viability of probiotic bacteria.

Probiotic food can be defined as "food containing live microorganisms believed to actively enhance health by improving the balance of microflora in the gut" (AFRC, 1989). Yoghurts have therapeutic benefits specially by incorporation of probiotic bacteria such as lactobacilli and bifidobacteria in dairy products (Lourens-Hattingh and Viljoen, 2001).

Shah (2000) says that the addition of ascorbic acid helped improve the survival and viability of $L$. acidophilus , so besides the health benefits, the high quantity of ascorbic acid of sea buckthorn have a positive influence on microorganisms and yogurt.

The purpose of this research was to optimize conventional technology scheme for obtaining yoghurt with added pulp of fruit in proportion of 3,5 , and $7 \%$ and sea buckthorn oil in proportion 1,2 and $3 \%$.

The relationship between major physicochemical parameters such as acidity, $\mathrm{pH}$ and lactic acid was graphically represented, in order to select the most stable probiotic yogurt .

Vol. XVII (2013), no. 1 


\section{MATERIALS AND METHODS}

Coding of samples: Samples were coded according to the addition of bioactive components: sea buckthorn oil, sea buckthorn pulp. Samples with dded oil were respectively noted with U, U1-1\%, U2-2\%, U3-3\%, percentage oil which was added. Samples with sea buckthorn pulp were noted with $\mathrm{K}, \mathrm{K} 1$ respectively for $3 \%, \mathrm{~K} 2$ - addition of $5 \%, \mathrm{~K} 3$-addition of $7 \%$.

Sea buckthorn : Sea buckthorn sample was purchased from SC NP Prod SRL, located in Marpod ,Sibiu county. The pulp of sea buckthorn obtained through centrifugal procedures is about 67\% (Heilscher and Lorber 1996).Oil of sea buckthorn was obtained from SC Hofigal SRL.

Making yoghurt: was performed by preparing probiotic starter culture that must be done under rigorous conditions of hygiene. The milk for yogurt was reated in a special preliminar and mechanical treatment because the curd obtained must be delicate. The oil and pulp of sea buckthorn was added after fermentation. Yoghurts were immediately cooled after fermentation and stored at $4{ }^{\circ} \mathrm{C}$ for 28 days.

Determination of acidity. This was done according to ISO 11869:2000, a certain amount of yoghurt was titrated with sodium hydroxide solution $0.1 \mathrm{~N}$ in the presence of phenolphthalein as indicator, until the sudden transfer of persistent pink color for 30 seconds.

$\mathrm{pH}$ determination. The $\mathrm{pH}$ of yoghurts was measured at termination $12 \mathrm{~h}$ post-fermentation, and subsequently at weekly intervals for 28 days.This was made according to STAS 8201-82, the end was performed using a $\mathrm{pH}$ meter (model HI 8010).

Determination of lactic acid: this was done according to instructions from R-Biopharm kit Cat method. No. 10139084035 spectrometry method using a model spectoforometrica STAT FAX 1904. The principle of the method consists in oxidation of L-lactic acid (L-lactate) to pyruvate by nicotinamide adenine dinucleotide (NAD), in the presence of L-lactate dehydrogenase (LDH -L) followed by a subsequent reaction catalyzed by the enzyme glutamate-pyruvate transaminase (GPT) in the presence of L-glutamate, the amount of $\mathrm{NADH}$ is determined by measuring the light absorbance at 340 nm using spectrometry STAT FAX 1904 model.

Statistical analysis: The data presented are the mean values \pm SD (Standard Deviation) of the three replicates. The statistical significance of differences

Vol. XVII (2013), no. 1 
among samples was determined by ANOVA Test. Differences in mean values was considered significantly different when $\mathrm{p}<0.05$.

Achievement graphs:The 3D Colormap Surface plot generated from XYZ data from a worksheet, where the data has uneven spacing in $\mathrm{X}$ and $\mathrm{Y}$. The raw data has been added as a 3D Scatter plot, and the symbols have been assigned the same color map as the surface.

\section{RESULTS AND DISCUSSIONS}

Titrable acidity, $\mathrm{pH}$ and lactic acid for the test sample were determined over a period of 28 days, measuring the titratable acidity at a 7 days interval from the first day.

\section{Variation of titratable acidity}

Changes in titratable acidity for yoghurt with added sea buckthorn oil are showed in Table 1, whereas the same variations in yoghurt with added sea buckthorn pulp is presented in Table 2. The samples inoculated with probiotic cultures have titratable acidity varying between 85 to $140{ }^{0} \mathrm{~T}$ from the first day of inoculation until day 28 for U1 sample with $1 \mathrm{ml}$ of sea buckthorn oil. The titrable acidity $90^{\circ} \mathrm{T}$ up at $153{ }^{0} \mathrm{~T}$ from day 0 to day 28 was inregistred for U2 sample with $2 \mathrm{ml}$ of sea buckthorn oil and U3 sample with values between 97 to $160{ }^{0} \mathrm{~T}$ from final day to day 0 . Compared with the values obtained for the blank (no added sea buckthorn oil) sea buckthorn oil sample values were lower and therefore the addition of sea buckthorn oil influences the fermentation process and the development of lactic cultures. The results include also the natural acidity of the milk and the developed acidity arising from bacterial activity. Titratable acidity may be a reasonable indication of the performance of the starter culture as shown the results similar with the results obtain by Tamime and Robinson (1999).

\section{Variation of $\mathbf{p H}$}

The results obtained at the analysis of $\mathrm{pH}$ variation are presented in Tables 3 and 4. $\mathrm{pH}$ variation for samples of yoghurt and probiotic cultures with sea buckthorn pulp had lower values compared to samples with higher added Sea Buckthorn as follows: K1 sample originally had reached $4.6 \mathrm{pH}$ that dropped to 4.3 on day 28 , the sample $\mathrm{K} 2$ originally had remained constant $4.3,14$

Vol. XVII (2013), no. 1 
days after the day 28 decreased to 4 and $\mathrm{K} 3$ sample initially had a $\mathrm{pH}$ of 3.9 reached after a period of 28 days to 3.8 i.e. the lowest value of all samples. Compared with the control sample which was initially 4.6 and finally 4.2 it can be seen that the addition of sea buckthorn pulp influenced the $\mathrm{pH}$ by a greater decrease in samples containing higher Sea Buckthorn similarly as acidity.

In samples of yoghurt with probiotic cultures and added sea buckthorn oil, variations characterized by a slight increase and then decrease were obtained as follows: U1 sample originally had a $\mathrm{pH}$ of 4.3 and then increased at day 14 remained constant at 4.6 weeks and again at day 28 decreased the value of 4.1; U2 initial sample $\mathrm{pH}$ was 4.2 with a slight increase in the next 14 days to 4.5 and then a sudden drop in on 28 for 4 . In the case of U3 sample, if the initial sample had a $\mathrm{pH}$ of 4.1 to 4.3 increased after 7 days and remained constant throughout the period, compared with the control sample that initially had $\mathrm{pH}$ of 4.6 decreased to 4.3 throughout the period. Increases in $\mathrm{pH}$ during storage period of 28 days could be due to buckthorn oil, compared with the control fermentation processes where $\mathrm{pH}$ decreases.

\section{Variation of lactic acid}

Variation of lactic acid was determined is presented in Tables 5 and 6. If U1 lactic acid sample has values between $9-7.63 \mathrm{~g} / 100 \mathrm{~g}$ of product from the first day until day 28 compared with lactic acid sample U3 where minimum values are between 0.33-2.9 g /100 g sample and the control sample without addition of sea buckthorn oil, in which sample values are approximately similar with U1. As can be seen the addition of seabuckthorn oil increased lactic acid and this oil had a negatively impact.

The addition of sea buckthorn oil negatively affect growth of lactic acid content similar to yoghurt from probiotic culture.

The addition of Sea Buckthorn pulp positively influence the enrichment of lactic acid content from yogurt with probiotic culture. Increases in lactic acid content may be due to a small extent of sea buckthorn pulp due to addition of organic acids.

In the samples of yoghurt with probiotic cultures and added sea buckthorn oil, variations characterized by a slight increase and then decrease were obtained. U1 sample originally had a $\mathrm{pH}$ of 4.3 and then increased at day 14 remained constant at 4.6 weeks and again at day 28 decreased the value of 4.1, U2 initial sample $\mathrm{pH}$ was 4.2 with a slight increase in the next 14 days to 4.5 and then a sudden drop in on 28 for 4.

Acta Universitatis Cibiniensis Series E: FOOD TECHNOLOGY

Vol. XVII (2013), no. 1 


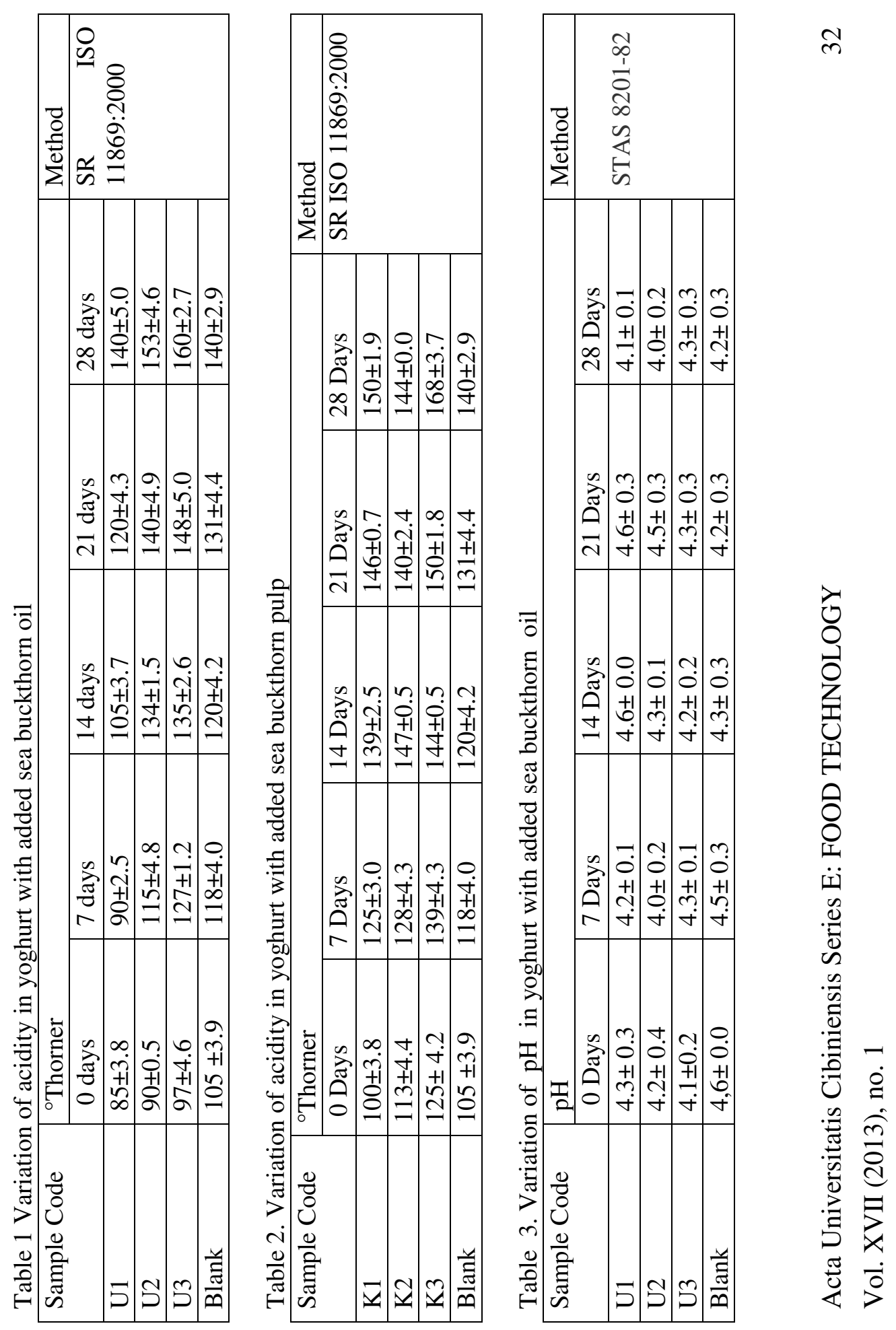




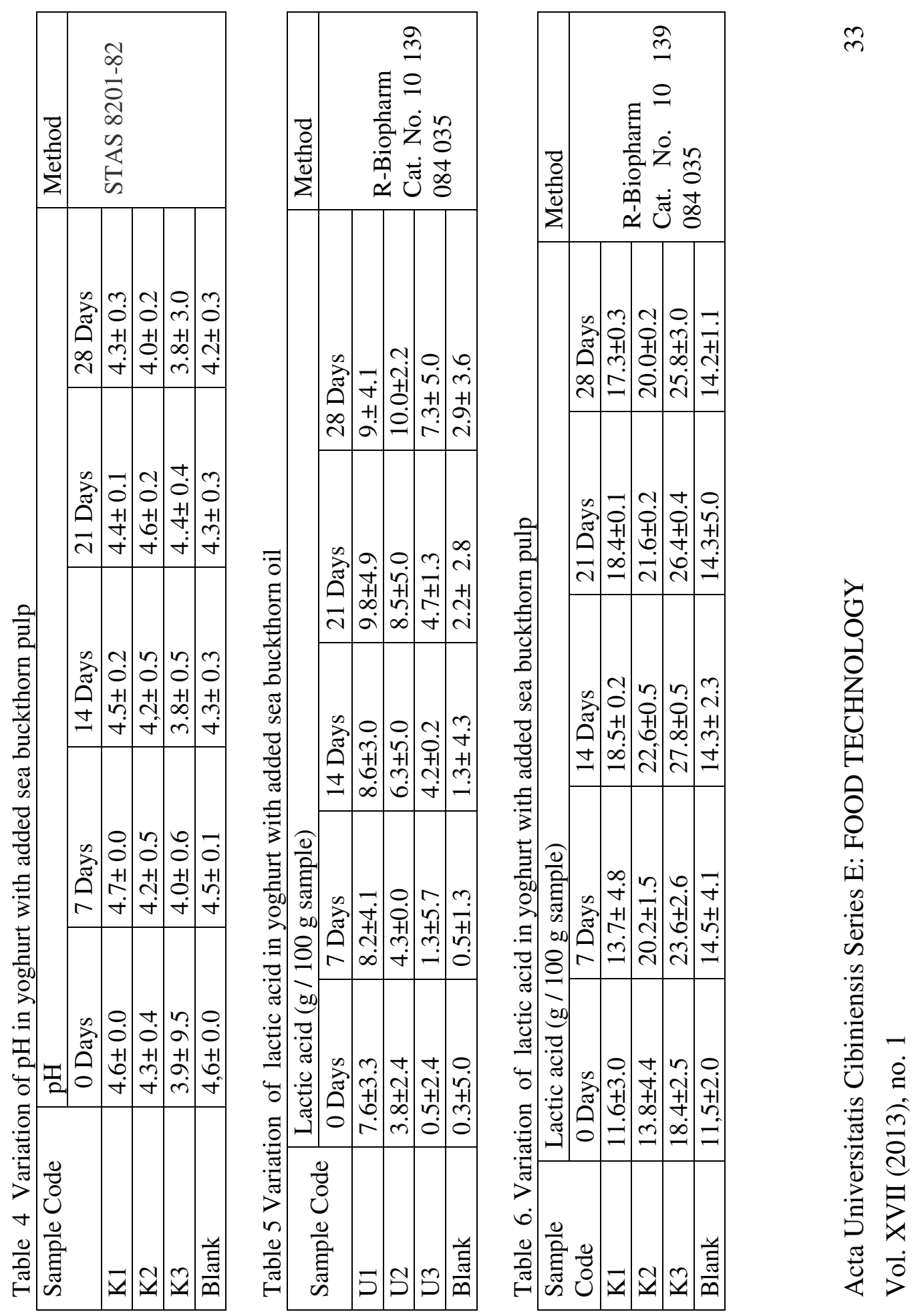


In the case of U3, if the initial sample had a pH of 4.1 to 4.3 increased after 7 days and remained constant throughout the period, compared with the control sample that initially had $\mathrm{pH}$ of 4.6 decreased to 4.3 throughout the period. Increases in $\mathrm{pH}$ during storage period of 28 days due to take back buckthorn oil, compared with the control fermentation processes where $\mathrm{pH}$ decreases.

The combined action of all three agents was represented graphically in Figures 1 and 2. pH seems to be the most crucial factor for the survival of $L$. acidophilus culture, as (Shah 2000) observed, also. Interpretation showed statistical significant differences between samples of probiotic yoghurt on the addition of oil and pulp of sea buckthorn compared with blank yoghurt. From the point of view of $\mathrm{pH}$, the optimum yoghurt sample was with added probiotic cultures obtained with oil in various proportions. During yoghurt manufacture, the $\mathrm{pH}$ of RSM declined from 6.50 to 4.50 for both batches when the gel was formed and fermentation was terminated at an incubation time of 3.50 and $4.55 \mathrm{~h}$ for probiotic and blank yoghurt respectively.

The $\mathrm{pH}$ of blank yoghurt increased from 4.50 to 4.55 at $12 \mathrm{~h}$ of storage at 4 ${ }^{\circ} \mathrm{C}$ and then declined slightly during cold storage, effect commonly known as 'post acidification.' A similar trend was observed for probiotic yogurt. The decline in $\mathrm{pH}$ after $12 \mathrm{~h}$ storage for both batches $(\mathrm{P}<0.05)$ varied between 4.45 and 4.21 for the probiotic yoghurt and 4.55 and 4.35 for the control yoghurt at the end of storage.

Lactic acid variation is observed in the smallest amount with added 3\% sea buckthorn pulp. The plot shows the acidity range for probiotic yoghurt with added bioactive components, since it increases as the amount of lactic acid is higher, but the $\mathrm{pH}$ will decrease. ANOVA-test showed that there are significant differences between blank yoghurt and probiotic culture samples, the same differences as significant probiotic yoghurt with added bioactive product in the sea buckthorn.

\section{CONCLUSIONS}

To improve the sensorial and nutritional quality of the yoghurt, the sea buckthorn was added in proportion of $3 \%$ pulp and oil $1 \%$, respectively. A higher percentage of the sea buckthorn pulp and oil may affect the sensorial quality also physicochemical parameters of yoghurt. Acidity increase the addition of bioactive compounds in various forms (oil or pulp).

Vol. XVII (2013), no. 1 

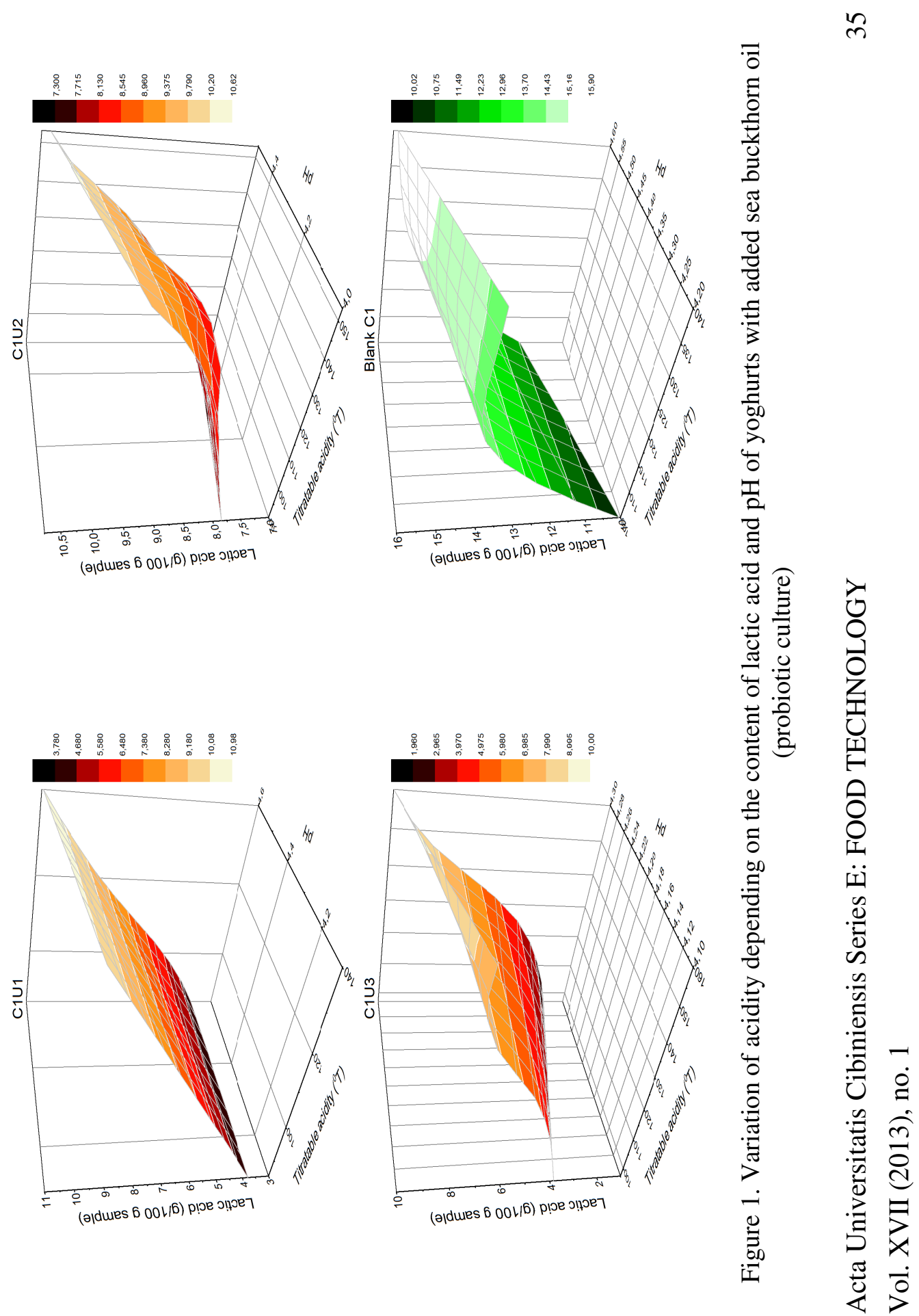


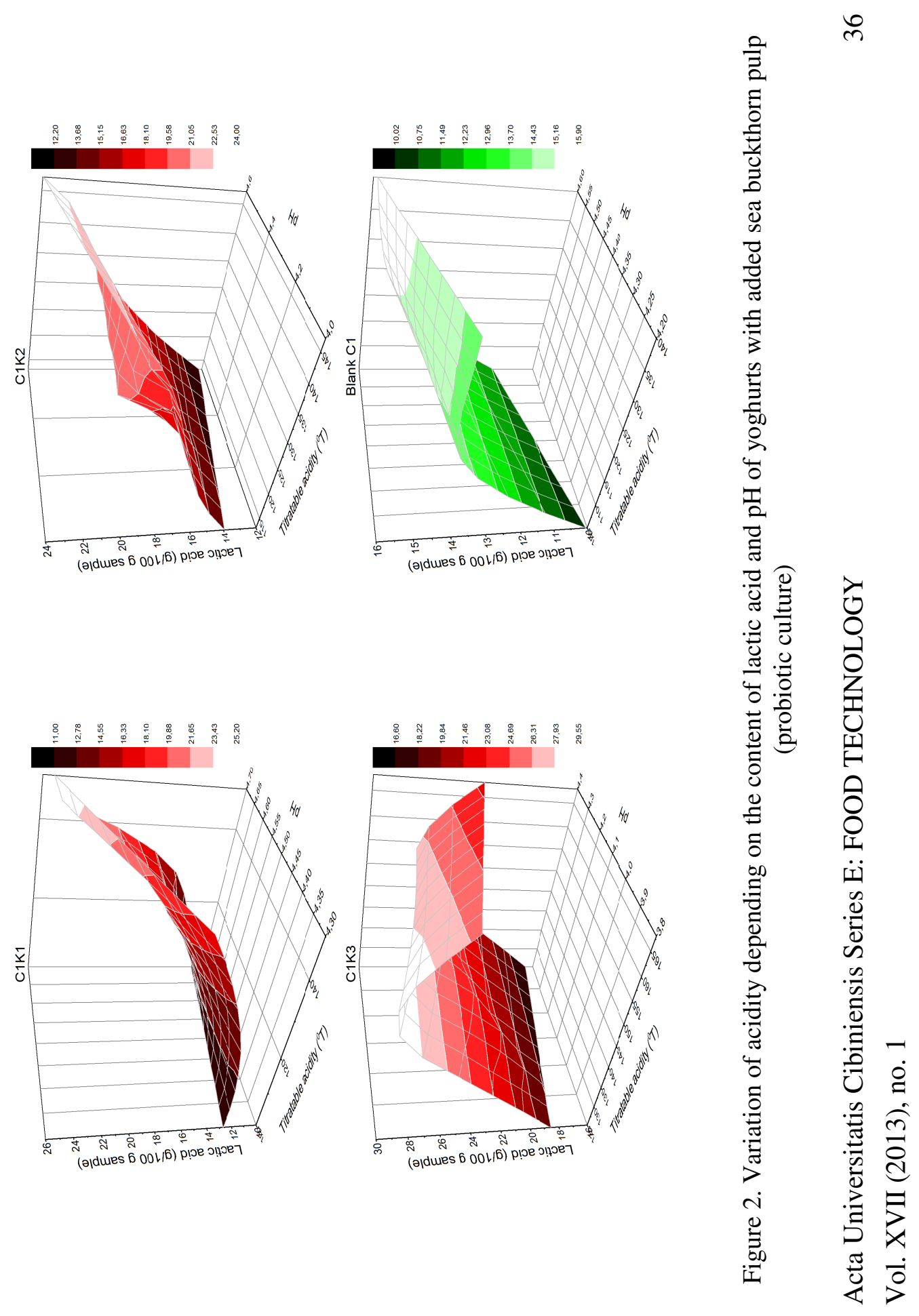


Yoghurt with added pulp of sea buckthorn obtained with probiotic culture has the highest acidity compared with other samples. On its period of validity increased acidity trend in the first 21 days and the last day until day of 28 did not increase significantly, but consistently.

The highest amount of accumulated lactic acid is registered in probiotic yoghurt with added 3\% sea buckthorn oil , and the lowest accumulated was record in probiotic yoghurt with added oil $1 \%$.

Regarding the investigated physicochemical parameters, the most stable yoghurt is that with addition of sea buckthorn pulp .

\section{REFERENCES}

1. AFRC R. F., Probiotics in man and animals, Journal of Applied Microbiology, 1989, 66 (5), 365-378

2. Beveridge T., Harrison J., Drover J., Processing effects on the composition of sea buckthorn juice from Hippophae rhamnoides L. Cv. Indian Summer, Journal of Agricultural and Food Chemistry, 2002, 50 (1), 113-116

3. Beveridge T., Li T. S., Oomah B. D., Smith A., Sea buckthorn products: manufacture and composition, Journal of agricultural and food chemistry, 1999, 47 (9), 3480-3488

4. Chen J., Effect of the immuno modulating agents (BCG) and the juice of HRL in the activity of splenic NK cells and LAK cells from tumour bearing mice, Chin J Microbiol Immunol, 1991, 11, 105-108

5. Goncharova N., Glushenkova A., Lipids of the leaves of two forms of central Asian sea buckthorn, Chemistry of natural compounds, 1996, 32 (4), 585-586

6. Hasler C. M., Functional foods: their role in disease prevention and health promotion, FOOD TECHNOLOGY-CHAMPAIGN THEN CHICAGO-, 1998, 52, 63-147

7. Heilscher K., Lorber S., Cold working process for obtaining clear juice, sediment and oil from sea buckthorn berries and their use, GFR Patent DE, 1996, 44 (31), 394

8. Karaaslan M., Ozden M., Vardin S., Turkoglu H., Phenolic fortification of yogurt using grape and callus extracts, LWT-Food Science and Technology, 2011, 44 (4), 1065-1072

9. Kris-Etherton P., Lefevre M., Beecher G.R., Gross M.D., Keen C.L., Etherton T.D., Bioactive compounds in nutrition and health-research

Vol. XVII (2013), no. 1 
methodologies for establishing biological function: the antioxidant and anti-inflammatory effects of flavonoids on atherosclerosis, Annu. Rev. Nutr., 2004, 24, 511-538

10. Lankaputhra W., Shah N., Improving viability of Lactobacillus acidophilus and bifidobacteria in yogurt using two step fermentation and neutralised mix, Food Australia, 1997, 49 (8), 363-366

11. Lourens-Hattingh A., Viljoen B. C., Yogurt as probiotic carrier food, International dairy journal, 2001, 11 (1), 1-17

12. Shah N., Probiotic bacteria: selective enumeration and survival in dairy foods, Journal of Dairy Science, 2000, 83 (4), 894-907

13. Tamime A. Y., Robinson R. K., Yoghurt: science and technology, Woodhead Publishing, 1999

Vol. XVII (2013), no. 1 